\title{
Radon-Wigner display: a compact optical implementation with a single varifocal lens
}

\author{
Sergio Granieri, Walter D. Furlan, Genaro Saavedra, and Pedro Andrés
}

\begin{abstract}
A new optical implementation of the Radon-Wigner display for one-dimensional objects is presented, making use of the fractional Fourier transform approach. The proposed setup makes use of only two conventional refractive elements: a cylindrical lens and a varifocal lens. Although the exact magnifications cannot be achieved simultaneously for all the fractional transforms, an optimum design can be obtained through balancing the conflicting magnification requirements. Experimental results are obtained with a commercially available progressive addition lens. For comparison, computer simulations are also provided. (C) 1997 Optical Society of America

Key words: Optical information processing, fractional Fourier transform, Wigner distribution function, Radon-Wigner transform, optical wave propagation.
\end{abstract}

\section{Introduction}

The Radon-Wigner transform (RWT) of a signal $t_{0}(x)$, defined as the projection of the Wigner distribution function of $t_{0}(x)$ at arbitrary angles in the phasespace domain, has been demonstrated to be useful in signal processing for the analysis and synthesis of multicomponent linear FM signals ${ }^{1,2}$ and for optimally detecting chirp signals in white Gaussian noise. ${ }^{3}$ For one-dimensional (1-D) signals it is possible to obtain a single two-dimensional display that contains a continuous representation of the RWT for all possible projection angles. This display is known as the Radon-Wigner spectrum ${ }^{4}$ or the RadonWigner display. ${ }^{5} \quad$ A relation between the RWT and another new important transformation, the fractional Fourier transform (FRT), ${ }^{6}$ was found by Lohmann and Soffer. ${ }^{7}$ They demonstrated that the RWT is the square modulus of the FRT, in which the fractional order $p$ is related to the projection angle $\phi$ of the RWT through the relationship $p=\phi / 90^{\circ}$. This finding suggests that the optical computation of the RWT is possible directly from the input function, omitting the passage through its Wigner distribution function.

The authors are with the Departamento de Óptica, Universitat de València, E-46100 Burjassot, Spain. The permanent address of S. Granieri is Centro de Investigaciones Ópticas (CIOp), C.C. 124, 1900 La Plata, Argentina.

Received 8 April 1997.

0003-6935/97/328363-07\$10.00/0

(C) 1997 Optical Society of America
Mendlovic et al. recently proposed an optical implementation of a discrete Radon-Wigner display using two multifaceted composite holograms. ${ }^{5}$ The resulting chart is limited to a finite number of angles (or equivalently to a finite number of fractional orders) because each hologram must be divided into a finite number of strips, each one coding a Fresnel zone plate with a different focal length.

We develop an optical system to obtain an approximated Radon-Wigner display, in which the fractional order varies continuously. The setup behaves like a multichannel parallel Radon-Wigner transformer and makes use of only two refractive elements: a cylindrical lens and a varifocal lens. In Section 2 we review some aspects of the optical production of the FRT, and we use them in the development of the optical system. The constraints for the involved parameters are also analyzed to achieve the optimum design. In Section 3 the performance of our proposal is tested experimentally with an ophthalmic progressive addition lens as a varifocal lens. These results are compared with computer simulations.

\section{Basic Theory}

Our approach for obtaining the Radon-Wigner display is through the FRT formalism with the use of the theorem demonstrated in Ref. 7. This approach is divided into two steps: first we review the optical production of the FRT in terms of Fresnel diffraction; next, we obtain in parallel the Radon-Wigner display for a 1-D input, by imaging simultaneously at the 
same output plane the FRT of all fractional orders between $p=0$ and $p=1$.

\section{A. Fractional Fourier Transform by Free Propagation}

The relationship between the FRT and the Fresnel diffraction was recognized by several authors. ${ }^{8-10}$ This connection can be understood clearly with the aid of Fig. 1, in which the classical Lohmann setup of type $\mathrm{II}^{6}$ is represented [Fig. 1(a)]. An equivalent configuration that uses free-space propagation is sketched in Fig. 1(b), in which the lens $L_{1}$ is replaced by a spherical wave front convergent to $S$, which coincides with the back focal point of $L_{1}$. In other words, the distance $z_{p}$ equals the focal length $f$ of both lenses. The amplitude outputs in these setups are, respectively, the FRT of order $p$ of the input amplitude $t\left(x_{0}, y_{0}\right)\left\{\Im^{p}\left[t\left(x_{0}, y_{0}\right)\right]\right\}$ and the Fresnel diffraction pattern of this input when it is illuminated with the spherical wave front $[U(x, y ; R)]$. Both results are related by

$$
\mathfrak{\Im}^{p}\left[t\left(x_{0}, y_{0}\right)\right]=\exp \left[i\left(\frac{k}{2 z_{p}}\right)\left(x^{2}+y^{2}\right)\right] U\left(x, y ; R_{p}\right),
$$

where

$$
\begin{aligned}
\Im^{p}\left[t\left(x_{0}, y_{0}\right)\right]= & \exp \left[\frac{i \pi}{\lambda f_{1}} \tan \left(p \frac{\pi}{2}\right)\left(x^{2}+y^{2}\right)\right] \\
& \times \iint t\left(x_{0}, y_{0}\right) \exp \left[\frac{i \pi}{\lambda f_{1}} \tan \left(p \frac{\pi}{2}\right)\right. \\
& \left.\times\left(x_{0}{ }^{2}+y_{0}{ }^{2}\right)\right] \exp \left[\frac{-2 i \pi}{\lambda f_{1}} \sin \left(p \frac{\pi}{2}\right)\right. \\
& \left.\times\left(x x_{0}+y y_{0}\right)\right] \mathrm{d} x_{0} \mathrm{~d} y_{0},
\end{aligned}
$$

being $f_{1}=f \tan \left(p \frac{\pi}{2}\right)$.

Therefore, aside from the quadratic phase factor introduced by the lens $L_{2}$, the FRT of order $p$ can be obtained by free propagation from the input transparency when it is illuminated with a spherical wave front whenever the distances in Fig. 1 are related by

$$
\begin{aligned}
& z_{p}=-f=\frac{-f_{1}}{\tan \left(p \frac{\pi}{4}\right)}, \\
& R_{p}=d=f_{1} \sin \left(p \frac{\pi}{2}\right) .
\end{aligned}
$$

The most important feature of the configuration of Fig. 1(b) is that for a fixed value of $z_{p}$, it provides not only the FRT of order $p$ at a distance $R_{p}$ from the input plane but also the scaled FRT's of all fractional orders located at different axial positions, shown as follows.

Let us assume that the distances in Fig. 1(b) are modified to achieve the FRT of order $q$ [these distances are obtained by replacing $p$ by $q$ in Eqs. (3) and (4)]. Using the Fresnel diffraction theory, we can (a)

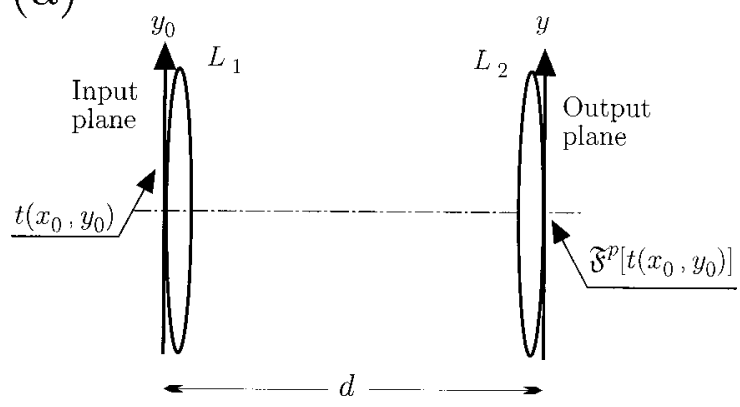

(b)

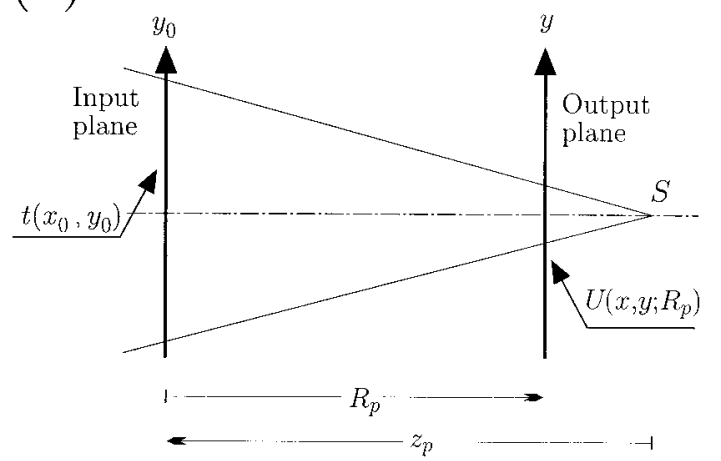

Fig. 1. Two equivalent setups to obtain the FRT of order $p$ : (a) Lohmann's setup of type II, (b) free-space propagation setup. The coordinates at the input and the output planes are $\left(x_{0}, y_{0}\right)$ and $(x, y)$, respectively. The correspondence between the distances in (a) and (b) are given through Eqs. (3) and (4).

recognize that if the input is placed at distance $z_{q} \neq$ $z_{p}$, the Fresnel diffraction pattern associated with the FRT of order $p$ is now achieved at a distance $R_{p}{ }^{\prime}\left(R_{p}{ }^{\prime}\right.$ $\neq R_{p}$ ) from the input with a magnification $M_{p}$, where $R_{p}{ }^{\prime}$ and $M_{p}$ can be obtained through the relations

$$
\begin{aligned}
\frac{1}{R_{p}{ }^{\prime}} & =\frac{1}{z_{p}}-\frac{1}{z_{q}}+\frac{1}{R_{p}}, \\
M_{p} & =\frac{R_{p}{ }^{\prime}}{R_{p}} .
\end{aligned}
$$

By replacing Eqs. (3) and (4) into Eqs. (5) and (6), we obtain

$$
\begin{aligned}
& R_{p}{ }^{\prime}=\frac{f_{1} \tan \left(p \frac{\pi}{2}\right)}{1-\frac{f_{1}}{z_{q}} \tan \left(p \frac{\pi}{2}\right)}, \\
& M_{p}=\frac{1+\tan \left(p \frac{\pi}{2}\right) \tan \left(p \frac{\pi}{4}\right)}{1-\frac{f_{1}}{z_{q}} \tan \left(p \frac{\pi}{2}\right)} .
\end{aligned}
$$


Therefore, given a fixed spherical convergent wave front, Eq. (7) allows us to achieve the axial position of the FRT of any arbitrary order. Moreover, this equation reveals that the FRT's of all fractional orders are axially distributed in a continuous way. In particular, all the fractional orders included in the interval $[0,1]$ are located between the input plane and the virtual point source $S$ [see Fig. 1(b)]. Each FRT is obtained, however, with a different scale factor given by Eq. (8).

\section{B. Radon-Wigner Display of a One-Dimensional Input}

Let us consider the optical arrangement sketched in Fig. 2 in which we assume a 1-D input $t\left(x_{0}\right)$. The 1-D nature of this signal permits us to work with a cylindrical wave front instead of the spherical one needed for two-dimensional inputs. Besides, because the FRT's present no variations along the vertical axis ( $y^{\prime}$-direction), we can select a different 1-D horizontal slice for use as a single fractional-order channel. In this way the FRT of a given fractionalorder $p$ can be accessed independently of the FRT's of the other fractional orders. The optical element $L$ in Fig. 2 should be designed to image the axially distributed FRT channels, with the proper magnification, at the same output plane simultaneously. As a consequence, for the Radon-Wigner display to be obtained, the focal length of this lens, $f$, should be different for each fractional-order $p$.

The setup of Fig. 2 takes advantage of the 1-D nature of the input and it behaves like a multichannel parallel FRT transformer, providing that the focal length of $L$ varies with $y^{\prime}$ in the same way as it varies with $p$. In this way the problem can be addressed as follows. For each value of $p$ (vertical coordinate $y^{\prime}$ ) we want to image a different object plane at a distance $a_{p}$ from the lens onto a fixed output plane located at $a^{\prime}$ from the lens. For obtaining this result, it is straightforward from the Gaussian lens equation that a lens with a focal length that varies with $p$ (vertical coordinate $y^{\prime}$ ) according to

$$
f(p)=\frac{a_{p} a^{\prime}}{a_{p}+a^{\prime}}
$$

is necessary. Taking into account (see Fig. 2) that

$$
a_{p}=l-R_{p}{ }^{\prime},
$$

we can rewrite Eq. (9) with Eq. (7) as

$$
f(p)=\frac{a^{\prime} l-f_{1} a^{\prime} \tan \left(p \frac{\pi}{2}\right)\left(1+\frac{l}{z}\right)}{a^{\prime}-l-f_{1} \tan \left(p \frac{\pi}{2}\right)\left(\frac{a^{\prime}+l+z}{z}\right)} .
$$

On the other hand, this focal length should provide the exact magnification at each output channel. The

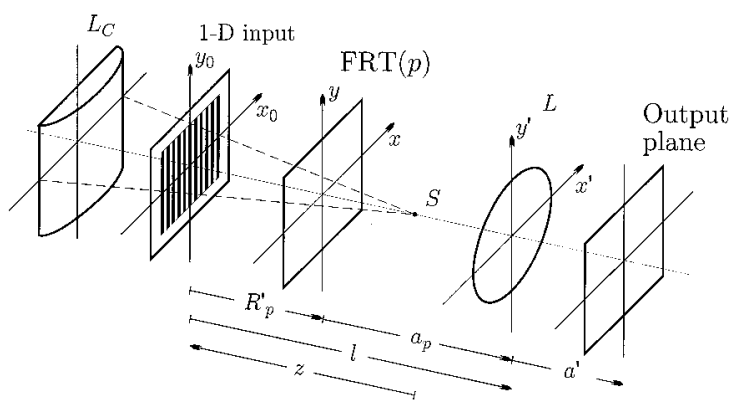

Fig. 2. Optical setup to obtain the Radon-Wigner display of 1-D inputs. The cylindrical lens $L_{C}$ provides the illuminating wavefront that converges to a line that crosses the optical axis at the point $S$. The free-space propagation provides the FRT's at distances $R^{\prime}$ from the input. Each one of such is imaged simultaneously by the varifocal lens $L$ at the output plane.

magnification given by the system for each fractionalorder $p$ is given by

$$
\begin{aligned}
M_{L}(p) & =\frac{-a^{\prime}}{a_{p}} \\
& =\frac{a^{\prime}}{R_{p}{ }^{\prime}-l} .
\end{aligned}
$$

Using Eq. (7), we obtain

$$
M_{L}(p)=\frac{a^{\prime}\left[-1+\frac{f_{1}}{z} \tan \left(p \frac{\pi}{2}\right)\right]}{l\left[1-\tan \left(p \frac{\pi}{2}\right)\left(\frac{f_{1}}{z}+\frac{f_{1}}{l}\right)\right]} .
$$

However, for the $p$-order slice of the RWT of the input function to be achieved, the lens $L$ should counterbalance the magnification of the FRT located at $R_{p}{ }^{\prime}$ to restore its proper magnification at the output

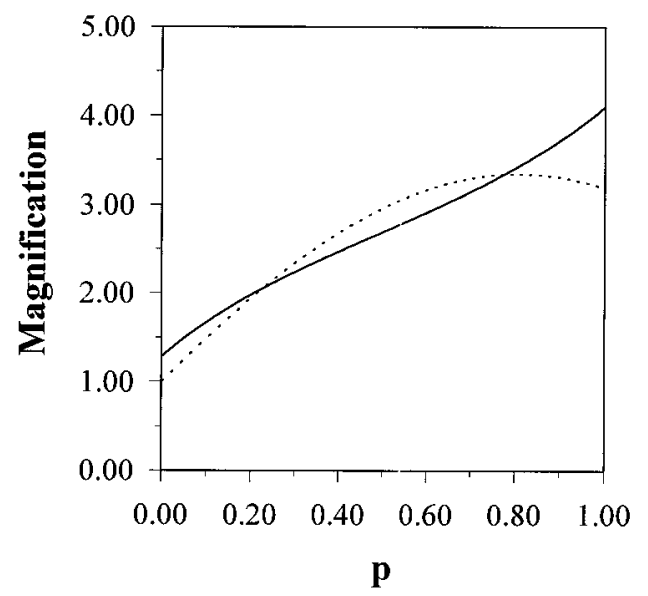

Fig. 3. Magnification provided by the lens $L$ in Fig. 2 for the values $z=426 \mathrm{~mm}, l=646 \mathrm{~mm}$, and $a^{\prime}=831 \mathrm{~mm}$, computed with Eq. (10) (approximated solution) in a solid curve and with Eq. (11) (exact solution) in a dotted curve. 
plane. Therefore, by using Eq. (8), we obtain that the magnification provided by $L$ should be

$$
M_{L}^{\prime}(p)=-\frac{1}{M_{p}}=\frac{-1+\frac{f_{1}}{z} \tan \left(p \frac{\pi}{2}\right)}{1+\tan \left(p \frac{\pi}{2}\right) \tan \left(p \frac{\pi}{4}\right)} .
$$

By comparing Eqs. (13) and (14), we note that the functional dependence of both equations on $p$ is different and, consequently, we are unable to obtain an exact solution for all fractional orders.

To obtain an approximated solution for our problem, we can select the four parameters of the system, namely, $z, l, f_{1}$, and $a^{\prime}$, in a way such that they minimize the difference between the functions defined by Eqs. (13) and (14) in the interval $[0,1]$. One way to find the values of such parameters is by a leastsquares method. To this end we define the merit function,

$$
\begin{aligned}
J\left(l, a^{\prime}, z\right)= & \int_{0}^{1}\left(\frac{M_{L}{ }^{\prime}-M_{L}}{M_{L}{ }^{\prime}}\right)^{2} \mathrm{~d} p \\
= & \frac{\pi}{2}\left(1+\frac{l f_{1}^{2}}{a^{\prime 2} z}\right)+\frac{\pi}{4} \frac{1}{a^{\prime 2}}\left(l+f_{1}^{2}+\frac{l^{2} f_{1}^{2}}{z^{2}}\right) \\
& +\frac{1}{a^{\prime 2}}\left(2 l-2 f_{1}-\frac{l f_{1}}{a^{\prime}}-\frac{2 l f_{1}}{z^{\prime}}-\frac{l^{2} f_{1}}{a^{\prime} z}\right),
\end{aligned}
$$

that should be minimized for achieving a minimum overall deviation between both results. This optimization leads to the following constraint conditions:

$$
\begin{aligned}
a^{\prime} & =l\left(\frac{1}{2}+\frac{\pi}{4}\right), \\
z & =\frac{-l f_{1}}{l+f_{1}} .
\end{aligned}
$$

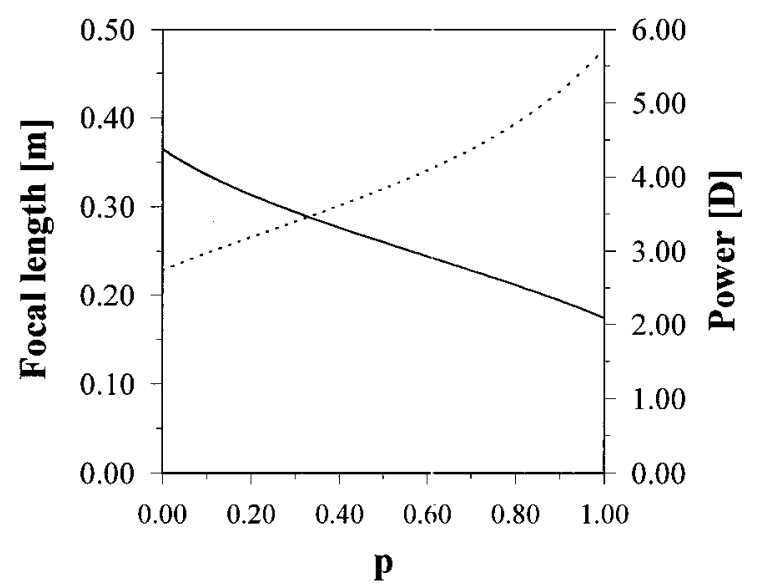

Fig. 4. Focal length (solid curve) and optical power (dotted curve) of the designed varifocal lens for values of $z, l$, and $a^{\prime}$ the same as in Fig. 3.
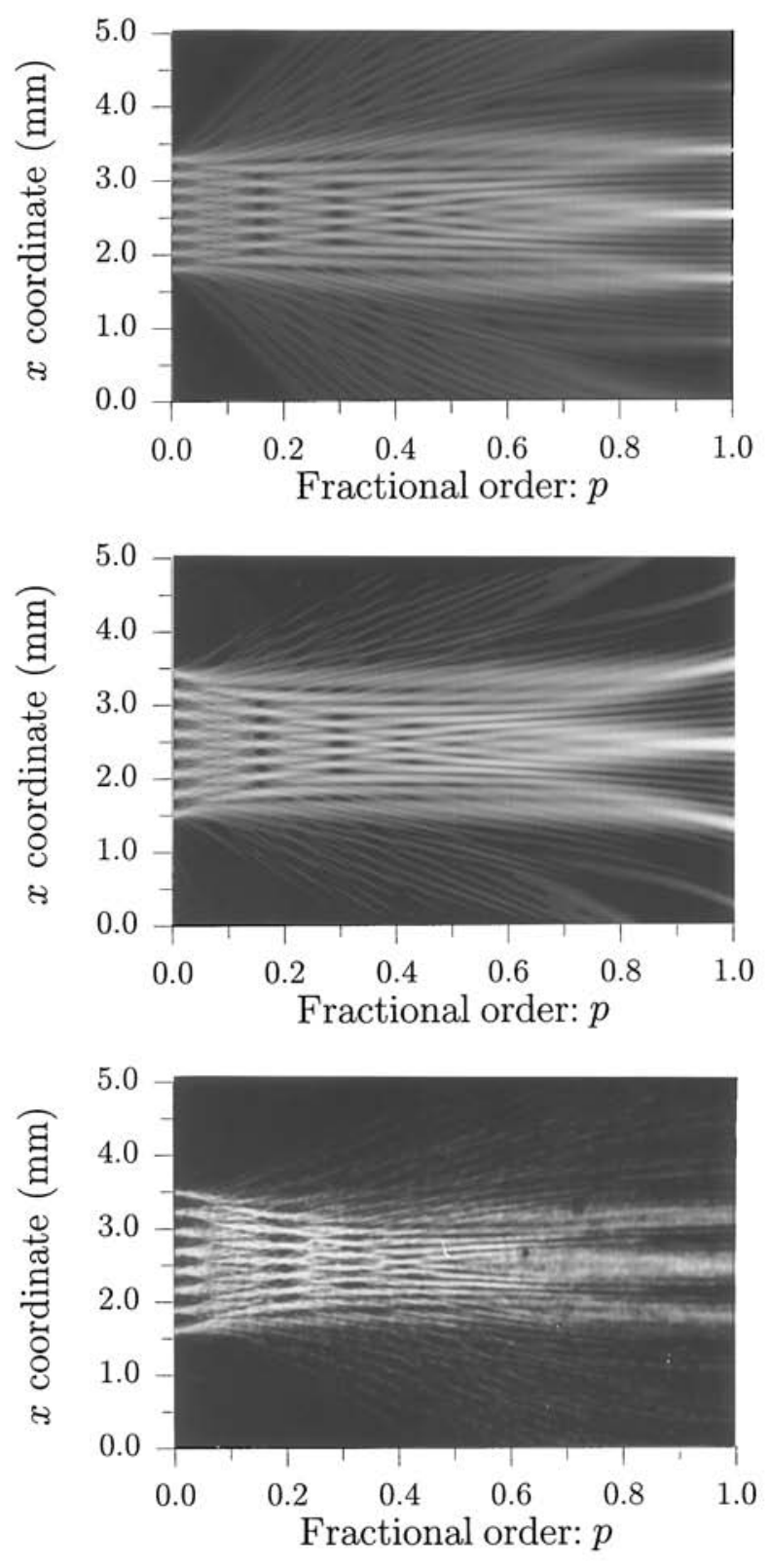

Fig. 5. Radon-Wigner display of a Ronchi grating of 3 lines/mm: (a) exact numerical simulation, (b) numerical simulation with the approximation discussed in Section 2, (c) experimental result.

A plot of $M_{L}$ and $M_{L}{ }^{\prime}$ under these conditions is shown in Fig. 3. It can be seen that the mean relative difference between both functions for a particular set of the involved parameters is approximately $10 \%$. The RWT is frequently of great interest in an intermediate region between the extremes given by the input function (corresponding to $p=0$, at one end) and its Fourier transform (corresponding to $p=1$, at the other end). In this case the limits of the integral in Eq. (15) can be chosen to take into account only the desired region. As far as this interval is reduced, the magnitude of the approximation achieved through minimizing Eq. (15) is improved. 

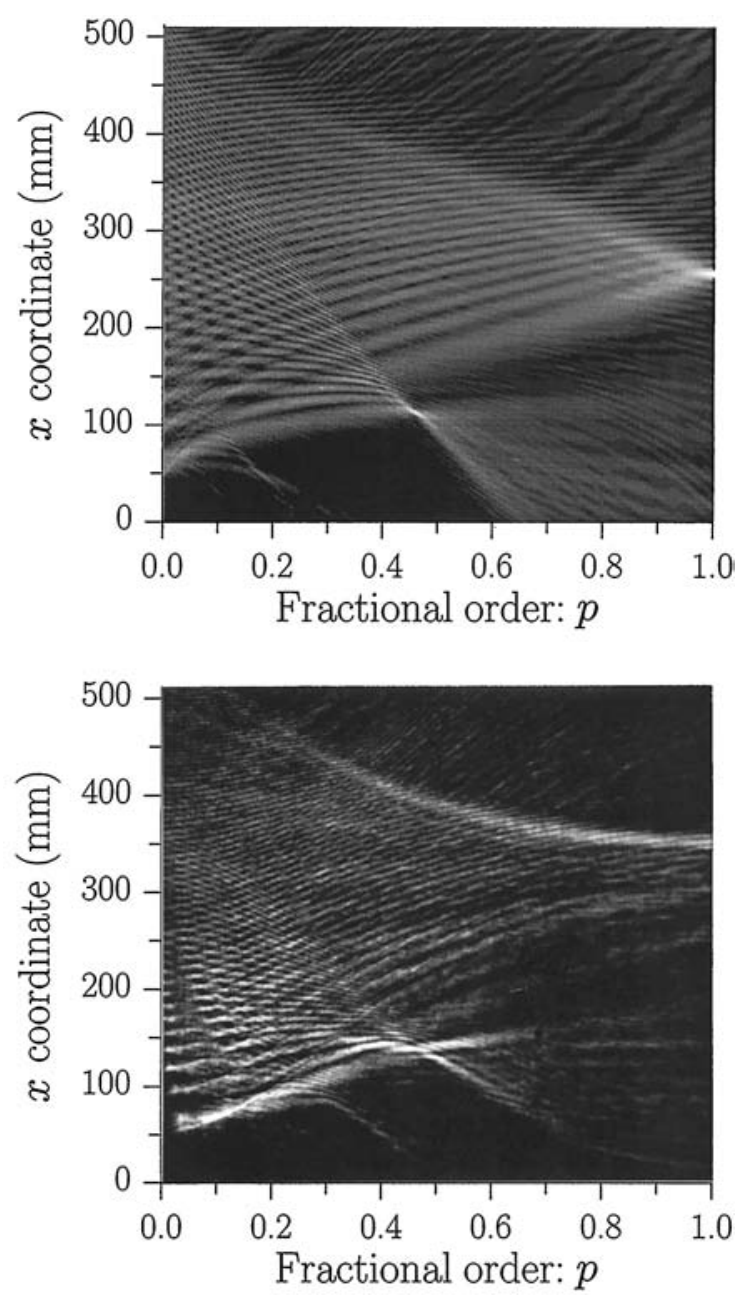

Fig. 6. Radon-Wigner display of a binary grating with a linearly increasing spatial frequency (chirp signal): (top) numerical simulation with the approximation discussed in Section 2, (bottom) experimental result.

\section{Experimental Results}

The variation of the focal distance of the lens $L$ with $p$ [Eq. (11)] and its optical power, under the constraints given by Eqs. (16) and (17), are represented in Fig. 4 for the following values: $z=426 \mathrm{~mm}, l=$ $646 \mathrm{~mm}$, and $a^{\prime}=831 \mathrm{~mm}$. It can be seen that for this particular combination of parameters the optical power is nearly linear except for values close to $p=1$. This linearity is also accomplished by ophthalmic progressive addition lenses in which there is a continuous linear transition between two optical powers that correspond to the so-called near portion and distance portion. The system of Fig. 2 was assembled with a progressive lens of $+2.75 \mathrm{D}$ spherical power and $+3 D$ of addition with the above-mentioned values of the parameters $z, l$, and $a^{\prime}$.

Figure 5 illustrates a comparison between the numerical simulations and the experimental results obtained with a Ronchi grating as input object. Figures 5(top) and 5(middle) are the numerical simulations of the RWT of the input computed exactly and with the approximation developed in Section 2,
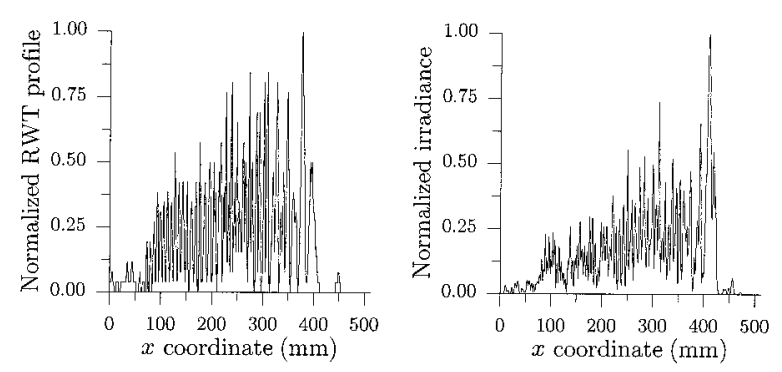

(a)
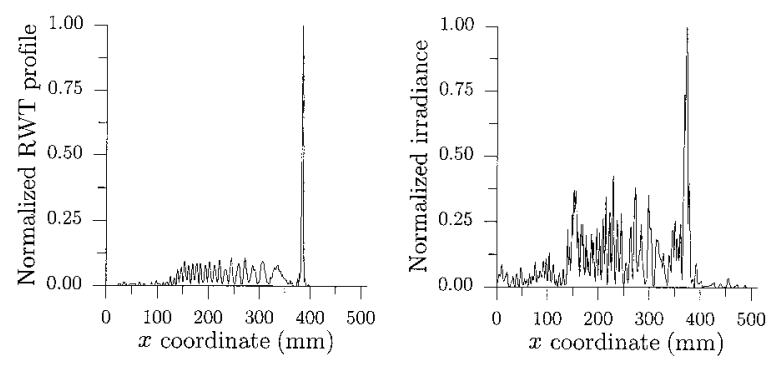

(b)
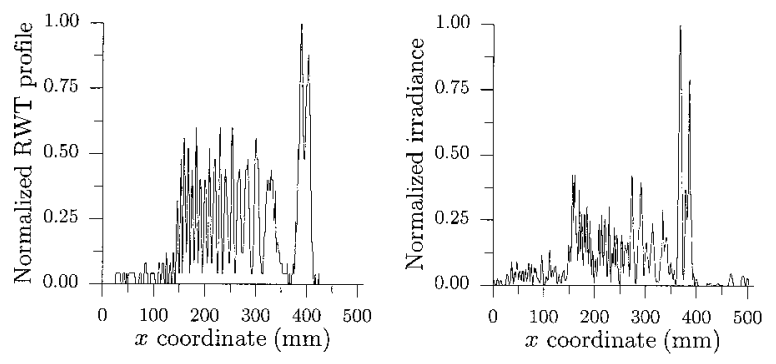

(c)

Fig. 7. Vertical profiles ( $p$ constant) obtained from Fig. 6 for three values of the fractional order $p$ : (a) $p=0.2$, (b) $p=0.45$, (c) $p=$ 0.5 . The figures on the left-hand side correspond to the numerical simulation [Fig. 6(top)], and those on the right-hand side correspond to the experimental data [Fig. 6(bottom)].

respectively. The experimental result is shown in Fig. 5(bottom). These figures confirm the validity of our proposal because only small differences in the magnification for the extreme values of $p$ can be appreciated (see also Fig. 3). The values of $p$ that correspond to the self-images (both positive and negative) can be identified clearly in the three views.

For a demonstration of the applicability of the proposed setup in practical techniques that make use of the RWT, we used it in a chirp-detection experiment. It is well known that this kind of signal generates an energy peak in the Radon-Wigner display. The $p$ coordinate of this maximum in the phase-space domain is directly related to the frequency-variation rate of the signal, i.e., the so-called chirp rate of the input function. Because the scale error in our proposal affects only the $x$ coordinate of the RadonWigner display, the chirp rate of an input chirp signal can be obtained from our device in an exact way, as it is performed from the true RWT. To test this possibility, we placed a binary grating with linearly increasing spatial frequency in the input plane of our 

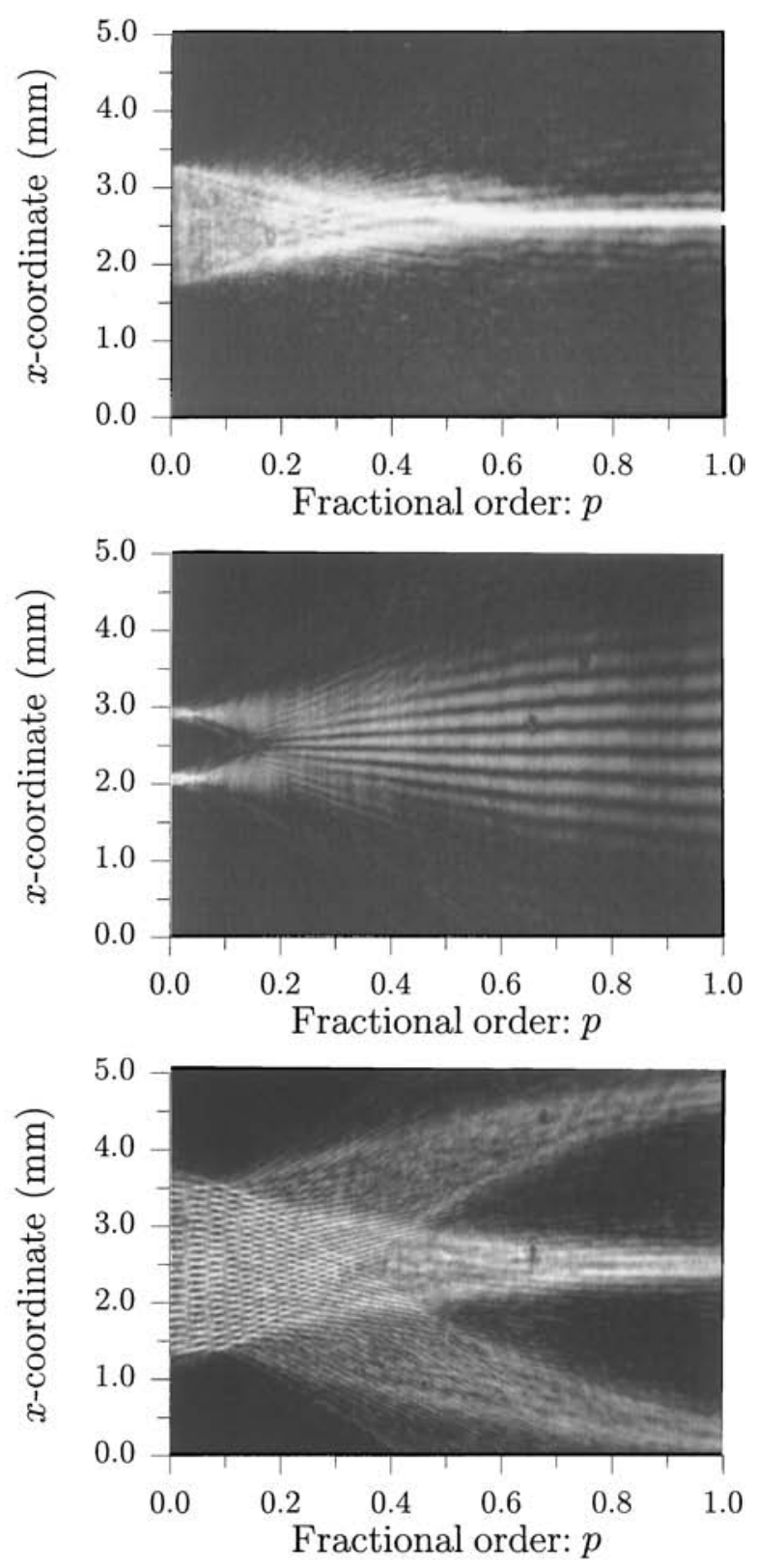

Fig. 8. Experimental results for the Radon-Wigner display: (top) single slit of $2.2 \mathrm{~mm}$, (middle) double slit of $0.23 \mathrm{~mm}$ separated by $1.05 \mathrm{~mm}$, (bottom) Ronchi grating of 7 lines $/ \mathrm{mm}$.

device. In Fig. 6 we present the experimental result obtained at the output plane of the setup together with the numerical simulation of the theoretical result presented in Section 2. From this later result we find the peak to be located at $p=0.45$.

Figure 7 shows three different profiles ( $p$ constant) for both the experimental and the computed data. In particular, in Fig. 7(b) the detection peak is clearly present in both cases. For smaller and larger values of $p$ this maximum vanishes, as is shown in Figs. 7(a) and $7(\mathrm{c})$, in a similar way in both cases. Two conventional parameters of the detection signals in Fig. 7(b) were used to compare both results quantitatively. First we computed the ratio of the peak en- ergy to the total energy in the profile [peak-to-total energy (PTE)]. This calculation provided the values of $\mathrm{PTE}=7 \%$ and $\mathrm{PTE}=2 \%$ for the theoretical and the experimental results, respectively. Second we calculated the ratio of the peak value to the highest secondary maximum in the detection profile [peakto-sidelobe ratio (PSLR)]. For this parameter we found PSLR $=9$ for the theoretical case and PSLR = 3 for the experimental data.

Finally, in Fig. 8 we illustrate the experimental Radon-Wigner display obtained for several test objects. Figures 8(top) and 8(middle) correspond to a single slit and a double slit, respectively, while Fig. 8(bottom) corresponds to a Ronchi grating with a spatial frequency of 7 lines $/ \mathrm{mm}$. These figures are quite interesting because they monitor the evolution of the diffraction patterns from the object plane to the Fraunhofer plane in a single display.

\section{Conclusions}

We implemented a simple optical setup for obtaining the Radon-Wigner display in which the fractional order is not limited to a discrete number of values as in previous proposals. The design strategy was based on the fact that the free-space propagation of a converging spherical wave front that illuminates an input transparency provides the FRT of this signal of all orders distributed along the optical axis. The components of the processor are conventional optical refractive elements such as cylindrical and varifocal lenses.

We present some experimental demonstrations of the capabilities of the setup, including an experience of detection of chirp signals. In all cases the agreement between the computer simulations and the experimental results confirms the good performance of the proposed system. These good results point to new possible applications of this device. For example, besides its use for describing the evolution of the complex amplitude of optical fields in free space and through a quadratic refractive index medium, ${ }^{11}$ the Radon-Wigner display can be used for implementing a multichannel parallel fractional Fourier correlator, which will be discussed in the future.

This study was supported by the Dirección General de Investigación Científica y Técnica (grant PB930354-C02-01), Ministerio de Educación y Ciencia. Sergio Granieri received support from the graduate fellowship program from Universitat de València. Walter Furlan thanks the Conselleria d'Educació i Ciència in the frame of the Plà Valencià de Ciència $i$ Tecnologia for financial support.

\section{References}

1. J. C. Wood and D. T. Barry, "Radon transformation of timefrequency distributions for analysis of multicomponent signals," Proc. IEEE Int. Conf. Acoust. Speech Signal Process. 4, 257-261 (1992).

2. J. C. Wood and D. T. Barry, "Linear signal synthesis using the Radon-Wigner transform," IEEE Trans. Signal Process. 42, 2105-2111 (1994). 
3. S. Kay and G. F. Boudreaux-Bartels, "On optimality of the Wigner distribution for detection," Proc. IEEE Int. Conf. Acoust. Speech Signal Process. 3, 1017-1020 (1985).

4. J. C. Wood and D. T. Barry, "Tomographic time-frequency analysis and its application toward time-varying filtering and adaptive kernel design for multicomponent linear-FM signals," IEEE Trans. Signal Process. 42, 20942104 (1994).

5. D. Mendlovic, R. G. Dorsch, A. W. Lohmann, Z. Zalevsky, and C. Ferreira, "Optical illustration of a varied fractional Fourier transform order and the Radon-Wigner display," Appl. Opt. 35, 3925-3929 (1996).

6. A. W. Lohmann, "Image rotation, Wigner rotation, and the frac- tional Fourier transform," J. Opt. Soc. Am. A 10, 2181-2186 (1993).

7. A. W. Lohmann and B. H. Soffer, "Relationships between the Radon-Wigner and fractional Fourier transforms," J. Opt. Soc. Am. A 11, 1798-1801 (1994).

8. P. Pellat-Finet, "Fresnel diffraction and the fractional-order Fourier transform," Opt. Lett. 19, 1388-1390 (1994).

9. H. M. Ozaktas and D. Mendlovic, "Fractional Fourier optics," J. Opt. Soc. Am. A 12, 743-751 (1995).

10. S. Granieri, O. Trabocchi, and E. E. Sicre, "Fractional Fourier transform applied to spatial filtering in the Fresnel domain," Opt. Commun. 119, 275-278 (1995).

11. T. Alieva and F. Agulló-Lopez, "Optical wave propagation of fractal fields,” Opt. Commun. 125, 267-274 (1996). 